\title{
Evaluation of Excitation Conditions of ITG Modes in the PANTA*)
}

\author{
Yudai MIWA, Naohiro KASUYA ${ }^{1,2)}$, Makoto SASAKI ${ }^{1,2)}$, Shigeru INAGAKI ${ }^{1,2)}$, \\ Kimitaka ITOH $^{2,3)}$, Masatoshi YAGI ${ }^{2,4)}$, Akihide FUJISAWA ${ }^{1,2)}$, Yoshihiko NAGASHIMA ${ }^{1,2)}$, \\ Maxime LESUR ${ }^{1,2)}$, Tatsuya KOBAYASHI, Soutarou YAMADA, Tetsuo YAMASHITA, \\ Satoshi OTSUBO, Shinsuke KAKIGAWA, Tomohiro MITSUZONO, \\ Hirofumi FUJINO and Sanae-I. ITOH ${ }^{1,2)}$ \\ Interdisciplinary Graduate School of Engineering Sciences, Kyushu University, Kasuga, Fukuoka 816-8580, Japan \\ ${ }^{1)}$ Research Institute for Applied Mechanics, Kyushu University, Kasuga, Fukuoka 816-8580, Japan \\ 2) Itoh Research Center for Plasma Turbulence, Kyushu University, Kasuga, Fukuoka 816-8580, Japan \\ 3) National Institute for Fusion Science, Toki, Gifu 509-5292, Japan \\ 4) Japan Atomic Energy Agency, Rokkasho, Aomori 039-3212, Japan
}

(Received 7 December 2012 / Accepted 10 May 2013)

To study the nonlinear saturation mechanism in turbulent plasmas, we plan to obseve ion temperature gradient (ITG) modes in the Plasma Assembly for Nonlinear Turbulence Analysis (PANTA). Linear growth rates of ITG modes in the linear device are analyzed to determine their excitation conditions using a three-field fluid model to describe the ion motion. Parameter scans of the linear growth rate show the threshold for ITG mode excitation, and the typical plasma parameters of the PANTA are found in the unstable domain.

(C) 2013 The Japan Society of Plasma Science and Nuclear Fusion Research

Keywords: ion temperature gradient mode, linear growth rate, linear device, threshold value, collision, local approximation, dispersion relation

DOI: $10.1585 /$ pfr. 8.2403133

\section{Introduction}

Understanding ion temperature gradient (ITG) modes ( $\eta_{\mathrm{i}}$ modes) is important because they represent a possible cause of anomalous ion energy transport in fusion plasmas [1-3]. To determine the fundamental properties of ITG modes, experiments and simulations have been carried out using the Columbia Linear Machine (CLM), which is a linear device. These experiments have shown an excitation of ITG instability, with a high parallel ITG $\nabla T_{\mathrm{i} \|}$ and a low density gradient $\nabla N$, that is the high $\eta_{\mathrm{i}}\left(=\nabla \ln T_{\mathrm{i} \|} / \nabla \ln N\right)$ [4]. The results have also been supported by numerical simulations [5]. For the Plasma Assembly for Nonlinear Turbulence Analysis (PANTA), detailed measurements by using multiple probe arrays have revealed structural formations with nonlinear mode coupling of unstable modes in resistive drift wave turbulence [6]. To study ITG turbulence, we plan to observe ITG modes and perform ion temperature measurements using ion-sensitive probes [7]. Predicting the excitation conditions of ITG modes is an urgent task for performing these observation.

As a first step, numerical analyses of linear growth rates are carried out using a fluid model. The set of model equations given in this study describes the ion motion with the effects of collisions. These equations are an extension of the Hamaguchi-Horton equation [8]. Parameter scans of the linear growth rate show the threshold for ITG mode

author'se-mail: yudai_miwa@riam.kyushu-u.ac.jp

*) This article is based on the presentation at the 22nd International Toki Conference (ITC22). excitation, and the dependences can be compared with our analytical solutions. Because the target plasma is collisional, the resistive drift wave instability is also important, whose numerical analyses have already been carried out [9]. In addition to that, the ITG instability that comes from ion motion may be one of the key players in plasma turbulence in the PANTA, which is studied in this paper.

This paper is organized as follows: In $\S 2$, the fluid model equations and plasma configuration are described. In $\$ 3$, the analytical solutions are explained, which show the tendency of ITG mode excitation. In $\$ 4$, the linear eigenmode analysis is performed to estimate excitation conditions of ITG modes in the PANTA. Finally, we summarize our results in $\$ 5$.

\section{Model of ITG Modes}

The ion time scale in the PANTA is in the range of $v_{\text {in }} \ll \Omega_{\mathrm{i}} \ll v_{\text {ii }}$, where $v_{\text {in }}$ is the ion-neutral collision frequency, $v_{\mathrm{ii}}$ is the ion-ion collision frequency, and $\Omega_{\mathrm{i}}$ is the ion cyclotron frequency. The plasma in the PANTA is collisional and has a much smaller mean free path than the ion Larmor radius; thus, a fluid model was adopted for the analyses.

\subsection{Fluid equations}

The model used for the analysis of ITG modes is described in this section. The set of model equations consists of the ion continuity equation, ion momentum conserva- 
tion equation, and ion energy conservation equation:

$$
\begin{aligned}
& \frac{\partial n_{\mathrm{i}}}{\partial t}+\nabla \cdot\left(n_{\mathrm{i}} \boldsymbol{v}_{\mathrm{i}}\right)=0, \\
& n_{\mathrm{i}} m_{\mathrm{i}} \frac{\mathrm{d} \boldsymbol{v}_{\mathrm{i}}}{\mathrm{d} t}=-\nabla p_{\mathrm{i}}+n_{\mathrm{i}} e\left(\boldsymbol{E}+\boldsymbol{v}_{\mathrm{i}} \times \boldsymbol{B}\right)+\boldsymbol{R}_{\mathrm{i}}, \\
& \frac{\mathrm{d} p_{\mathrm{i}}}{\mathrm{d} t}=-\gamma p_{\mathrm{i}} \nabla \cdot \boldsymbol{v}_{\mathrm{i}}-(\gamma-1) \nabla \cdot \boldsymbol{q}_{\mathrm{i}},
\end{aligned}
$$

where $p_{\mathrm{i}}$ is the ion pressure, $n_{\mathrm{i}}$ is the ion density, $m_{\mathrm{i}}$ is the ion mass, $v_{\mathrm{i}}$ is the ion velocity, $\gamma$ is the heat capacity ratio, $\boldsymbol{E}$ is the electric field, $\boldsymbol{R}_{\mathrm{i}}$ is the friction force, and $\boldsymbol{q}_{\mathrm{i}}$ is the heat flux. For simplicity, the Boltzmann relation and quasi-neutral conditions were assumed:

$$
\begin{aligned}
& n_{\mathrm{e}}=n_{\mathrm{i}}, \\
& n_{\mathrm{e}}=n_{0} \exp \left(e \phi / T_{\mathrm{e}}\right) .
\end{aligned}
$$

Note that the collision effect on an electron is not negligible in the PANTA; thus, excitation of the resistive drift wave is possible, which is not considered in this paper.

For simplicity, the following assumptions were introduced;

- Negligible heat flux : $\nabla \cdot \boldsymbol{q}_{\mathrm{i}}=0$ :

- Parallel friction force only:

$$
\boldsymbol{R}_{\mathrm{i}}=\boldsymbol{R}_{\mathrm{i} \|}=-n_{\mathrm{i}} m_{\mathrm{i}} v_{\mathrm{in}} \boldsymbol{v}_{\mathrm{i} \|},
$$

- Constant electron temperature : $T_{\mathrm{e}}=T_{\mathrm{e} 0}$ :

- Perpendicular ion velocity given by the $E \times B$ drift:

$$
\boldsymbol{v}_{\mathrm{i} \perp}=(\boldsymbol{B} \times \nabla \tilde{\phi}) / B^{2} .
$$

Because the target plasma has a rather low temperature, an effect of neutrals was included, which can weaken instability [9]. The finite Larmor radius effect and other kinetic effects were not included because the plasma is collisional. Each variable was rewritten into the following form for cylindrical coordinate:

$$
f=f_{\mathrm{eq}}(r)+\tilde{f}(r, \theta, z, t),
$$

where the first and second terms represent the equilibrium and fluctuation parts, respectively. In addition, $\phi=\tilde{\phi}, v_{\mathrm{i} \|}=$ $\tilde{\boldsymbol{v}}_{\mathrm{i} \|}$ and the Boltzmann relation $\tilde{n}=e \tilde{\phi} / T_{\mathrm{e}}$ were assumed. The linearized set of Eqs. (1-3) was then rewritten:

$$
\begin{gathered}
\frac{\partial}{\partial t}\left(1-\nabla_{\perp}^{2}\right) \tilde{\Phi}=-\nabla_{\|} \cdot \tilde{V}+\frac{\partial N_{\mathrm{eq}}}{\partial r} \frac{1}{r} \frac{\partial \tilde{\Phi}}{\partial \theta} \\
+\frac{\partial P_{\mathrm{eq}}}{\partial r} \frac{1}{r} \frac{\partial \nabla_{\perp}^{2} \tilde{\Phi}}{\partial \theta}-\mu_{W} \nabla_{\perp}^{4} \tilde{\Phi}, \\
\frac{\partial \tilde{V}}{\partial t}=-\nabla_{\|}(\tilde{P}+\tilde{\Phi})-v_{\mathrm{in}} \tilde{V}+\mu_{V} \nabla_{\perp}^{2} \tilde{V}, \\
\frac{\partial \tilde{P}}{\partial t}=-\gamma \theta_{\mathrm{i}} \nabla_{\|} \cdot \tilde{V}+\frac{\partial P_{\mathrm{eq}}}{\partial r} \frac{1}{r} \frac{\partial \tilde{\Phi}}{\partial \theta}+\mu_{P} \nabla_{\perp}^{2} \tilde{P} .
\end{gathered}
$$

Equation (7) is a combination of Eq. (1) and the rotation of the perpendicular part of Eq. (2). The following normalizations were introduced here;

$$
\begin{aligned}
& r=\frac{r}{\rho_{\mathrm{s}}}, \quad t=\Omega_{\mathrm{ci}} t, \quad \boldsymbol{v}_{\mathrm{i}}=\frac{\boldsymbol{v}_{\mathrm{i}}}{c_{\mathrm{s}}}, \quad \theta_{\mathrm{i}}=\frac{T_{\mathrm{i} 0}}{T_{\mathrm{e}}}, \\
& N=\ln \left(n_{\mathrm{i}}\right), \quad \Phi=\frac{e \phi}{T_{\mathrm{e}}}, \quad V=\frac{\boldsymbol{v}_{\mathrm{i} \|}}{c_{\mathrm{s}}}, \quad P=\frac{p_{\mathrm{i}}}{p_{\mathrm{e} 0}},
\end{aligned}
$$

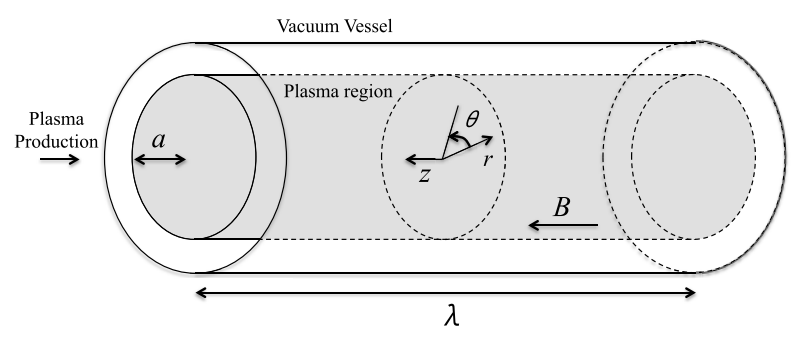

Fig. 1 Cylindrical plasma configuration.

where $\Omega_{\mathrm{ci}}=e B / m_{\mathrm{i}}$ is the ion cyclotron frequency, $c_{\mathrm{s}}=$ $\sqrt{T_{\mathrm{e}} / m_{\mathrm{i}}}$ is the ion sound velocity, and $\rho_{\mathrm{s}}=c_{\mathrm{s}} / \Omega_{\mathrm{ci}}$ is the effective Larmor radius (at the electron temperature). Viscosities $\mu_{W}, \mu_{V}$, and $\mu_{P}$ were added in to Eqs. (7-9) to account for dissipation effects. Furthermore, in Eq. (2), perpendicular to the magnetic field, an unperturbed part of a diamagnetic drift was accounted for;

$$
\boldsymbol{v}_{\mathrm{i} \perp}=(\boldsymbol{B} \times \nabla \tilde{\phi}) / B^{2}+\left(\boldsymbol{B} \times \nabla \tilde{p}_{\mathrm{i}, \mathrm{eq}}\right) / e n_{\mathrm{i}} B^{2} .
$$

This term is substantial to excite ITG instability [10]. This model consists of evolution equations for three fields $\Phi$, $V$ and $P$, which is an extension of the Hamaguchi-Horton equations [8].

\subsection{Plasma configuration}

The plasma configuration is shown in Fig. 1. The plasma has a simple cylindrical shape with device length $\lambda$, whose boundary is given at $r=a$. For simplicity, the vacuum region was not treated, and the magnetic field was only allowed to have an axial component, $\boldsymbol{B}=B \boldsymbol{z}=$ constant. The high-density, low-temperature plasma produced by helicon waves in a discharge of argon was simulated. Cylindrical coordinates $(r, \theta, z)$ were used for the model geometry, and the periodic boundary condition in the azimuthal $(\theta)$ and axial $(z)$ directions was considered to adopt the spectral expansion by Fourier transform;

$$
\tilde{f}=\tilde{f}_{m n}(r) \times \exp \left[i\left(-\omega_{m n} t+m \theta+\frac{2 \pi n}{\lambda}\right)\right] .
$$

The radial boundary condition was set to

$$
\tilde{f}_{m n}(r)=0 \quad(\text { at } r=0, a) .
$$

\section{Analytical Solutions}

Analytical solutions with a local approximation help us understand the excitation conditions of ITG modes [10]. The following Fourier representation was used:

$$
\tilde{f}=\tilde{f}_{k, \omega} \times \exp \left\{\mathrm{i}\left[-\omega t+k_{r} r+k_{\theta}(a \theta)+k_{z} z\right]\right\},
$$

where

$$
k_{r}=\frac{\pi l}{a}, \quad k_{\theta}=\frac{m}{a}, \quad k_{z}=\frac{2 \pi n}{\lambda},
$$


$\omega$ is a frequency, and $l, m$, and $n$ are the radial, azimuthal, and axial mode numbers, respectively. The set of Eqs. (79) gives the following dispersion relation in a dimensional form:

$$
\begin{array}{r}
\left(1+\rho_{\mathrm{s}}^{2} k_{\perp}^{2}\right) \omega^{2}-\left(1-\kappa_{\mathrm{i}} \rho_{\mathrm{s}}^{2} k_{\perp}^{2}\right) \omega_{* \mathrm{e}} \omega \\
-c_{\mathrm{s}}^{2} k_{z}^{2}\left[\frac{1+\kappa_{\mathrm{i}} \omega_{* \mathrm{e}} / \omega}{1-\gamma \theta_{\mathrm{i}} c_{\mathrm{s}}^{2} k_{z}^{2} / \omega^{2}}\right]=0,
\end{array}
$$

where $\omega_{* \mathrm{e}}=\left(\rho_{\mathrm{s}} / L_{N}\right) c_{\mathrm{s}} k_{\theta}, \kappa_{\mathrm{i}}=\theta_{\mathrm{i}}\left(1+\eta_{\mathrm{i}}\right)$, and $\eta_{\mathrm{i}}=L_{N} / L_{T \mathrm{i}}=$ $\nabla_{r} \ln T_{\mathrm{i}} / \nabla_{r} \ln N$. Here $\mu=v_{\text {in }}=0$ was assumed for simplicity. In limited cases, analytical solutions can be found: (i) when $|\omega| \ll\left|\omega_{* \mathrm{e}}\right|$,

$$
\omega= \pm c_{\mathrm{s}} k_{z} \sqrt{\gamma \theta_{\mathrm{i}}-\frac{\kappa_{\mathrm{i}}}{1-\kappa_{\mathrm{i}} \rho_{\mathrm{s}}^{2} k_{\perp}^{2}}},
$$

except when $\rho_{\mathrm{s}} k_{\perp} \sim \kappa_{\mathrm{i}}^{-1 / 2}$,

$$
\omega=\frac{-1 \pm i \sqrt{3}}{2} \omega_{+}+\frac{-1 \mp i \sqrt{3}}{2} \omega_{-},
$$

where

$$
\begin{aligned}
& \omega_{ \pm}=\left(-q \pm\left(q^{2}+p^{3}\right)^{1 / 2}\right)^{1 / 3}, \\
& q=-\frac{\kappa_{\mathrm{i}}^{2}}{2\left(1+\kappa_{\mathrm{i}}\right)} \omega_{* \mathrm{e}} k_{z}^{2} c_{\mathrm{s}}^{2}, \\
& p=-\frac{\gamma \theta_{\mathrm{i}} k_{z}^{2} c_{\mathrm{s}}^{2}}{3} .
\end{aligned}
$$

(ii) when $|\omega| \gg\left|\omega_{* \mathrm{e}}\right|$, it becomes

$$
\omega= \pm c_{\mathrm{s}} k_{z} \sqrt{\gamma \theta_{\mathrm{i}}+\frac{1}{1+\rho_{\mathrm{s}}^{2} k_{\perp}^{2}}} .
$$

Solution (16) becomes unstable when

$$
\kappa_{\mathrm{i}}>\frac{\gamma \theta_{\mathrm{i}}}{1+\gamma \theta_{\mathrm{i}} \rho_{\mathrm{s}}^{2} k_{\perp}^{2}} .
$$

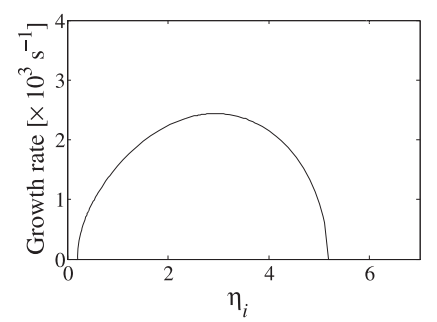

(a)

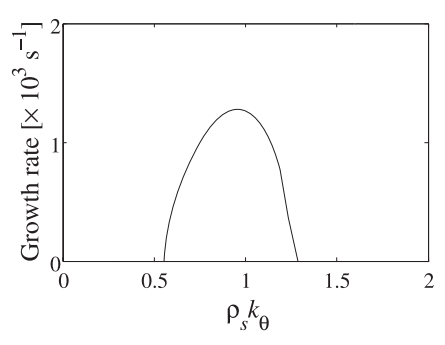

(b)

Fig. 2 Growth rate as a function of (a) $\eta_{\mathrm{i}}$ and (b) $\rho_{\mathrm{s}} k_{\theta}$
This condition gives $\eta_{\mathrm{i}}>2 / 3$, when $\rho_{\mathrm{s}} k_{\perp} \rightarrow 0$ and $\gamma=5 / 3$. The ITG modes become unstable when $\kappa_{\mathrm{i}}$ is larger than the threshold value. As $\kappa_{\mathrm{i}}$ increases, $|\omega|$ increases accordingly, and the $\nabla_{\|} \tilde{\Phi}$ term in Eq. (8) becomes effective, which stabilizes the mode. Therefore, there are upper limits in $\kappa_{\mathrm{i}}$ and $\eta_{\mathrm{i}}$. Then the modes are stable in the limit of a large frequency in Eq. (18). Figure 2 (a) shows the growth rate as a function of $\eta_{\mathrm{i}}$ with $(l, m, n)=(1,1,1)$, $\gamma=5 / 3, \theta_{\mathrm{i}}=0.1, \rho_{\mathrm{s}} k_{\perp}=1.84, c_{\mathrm{s}} k_{z}=4.1 \times 10^{3} \mathrm{~s}^{-1}$, and $\omega_{* \mathrm{e}}=28 \times 10^{3} \mathrm{~s}^{-1}$, which was obtained by solving Eq. (15).

The magnitude of $k_{\perp}$ also affects instability. Solution (16) becomes stable when $\rho_{\mathrm{s}} k_{\perp}$ becomes large. Even if $\gamma \theta_{\mathrm{i}}>\kappa_{\mathrm{i}}$, which is the case when the ITG modes are stable with $\rho_{\mathrm{s}} k_{\perp}=0$, the modes become unstable with $\rho_{\mathrm{s}} k_{\perp} \sim \kappa_{\mathrm{i}}^{-1 / 2}$, as is in Eq. (15). Therefore, there are upper and lower limits for the instability in $\rho_{\mathrm{s}} k_{\perp}$. Figure 2 (b) shows the growth rate as a function of $\rho_{\mathrm{s}} k_{\theta}$ with $(l, n)=$ $(1,1), \gamma=5 / 3, \theta_{\mathrm{i}}=0.1, \eta_{\mathrm{i}}=0.2, c_{\mathrm{s}}=2.6 \times 10^{3} \mathrm{~m} / \mathrm{s}$, $k_{z}=1.58 \mathrm{~m}^{-1}$, and $L_{N}=50 \mathrm{~mm}$.

In a drift wave mechanism, parallel motion connects the density and the potential, and therefore, it needs the finite $k_{z}$. Modes with a larger $k_{z}$ become stable because a charge separation is canceled more easily by a larger $E_{z}$.

\section{Excitation Condition \\ 4.1 Plasma parameters}

The eigenvalue problem was solved using the set of differential equations shown in Eqs. (7-9). The parameters were selected to simulate the PANTA: argon plasma, $B=0.04 \mathrm{~T}, T_{\mathrm{e}}=3.0 \mathrm{eV}, T_{\mathrm{i} 0}=0.3 \mathrm{eV}, a=5.0 \mathrm{~cm}$, and $\lambda$ $=4.0 \mathrm{~m}$. Using these parameters, the ion cyclotron frequency is $\Omega_{\mathrm{ci}} / 2 \pi=15 \mathrm{kHz}$, the ion acoustic velocity is $c_{\mathrm{s}}$ $=2.6 \times 10^{3} \mathrm{~m} / \mathrm{s}$, the effective Larmor radius is $\rho_{\mathrm{s}}=28 \mathrm{~mm}$ and the mass ratio $m_{\mathrm{i}} / m_{\mathrm{e}}=7.3 \times 10^{4}$. The mean free pass is $\lambda_{\mathrm{mfp}}=\rho_{\mathrm{i}} \Omega_{\mathrm{ci}} / v_{\mathrm{ii}}=0.2 \mathrm{~mm}$, which was given by the ionion collision frequency $v_{\mathrm{ii}} \sim 40 \Omega_{\mathrm{ci}}$, and is smaller than the ion Larmor radius. The profile of equilibrium density, ion temperature, and ion pressure profiles were assumed to be

$$
\begin{aligned}
& N_{\mathrm{eq}}(r)=N_{0} \exp \left[-\left(r / L_{N}\right)^{2}\right], \\
& T_{\mathrm{i} \text { eq }}(r)=T_{\mathrm{i} 0} \exp \left[-\left(r / L_{T \mathrm{i}}\right)^{2}\right], \\
& P_{\mathrm{eq}}(r)=P_{0} \exp \left[-\left(r / L_{P}\right)^{2}\right],
\end{aligned}
$$

respectively, where

$$
L_{P}=L_{N}\left(1+\eta_{\mathrm{i}}\right)=\frac{L_{N} L_{T \mathrm{i}}}{L_{N}+L_{T \mathrm{i}}} .
$$

Each parameter was chosen so that $L_{N}=50 \mathrm{~mm}, L_{T \mathrm{i}}=$ $250 \mathrm{~mm}, \eta_{\mathrm{i}}=0.2, N_{0}=1.0$, and $P_{0}=T_{\mathrm{i} 0} / T_{\mathrm{e}}=0.1$.

The ion-ion collision frequency There is no precise measurement of the neutral density in the PANTA. A rough estimation of the ion-neutral collision was used:

$$
v_{\text {in }}=\frac{1-\alpha}{\alpha} n_{\mathrm{i} 0} \sigma_{\text {in }}\left|v_{\mathrm{i}}\right| \sim 0.05 \Omega_{\mathrm{ci}},
$$

where an average density $n_{\mathrm{i} 0}=10^{19} \mathrm{~m}^{-3}$, an ionization rate 
$\alpha=0.5$, and a cross section for ion-neutral collision $\sigma_{\text {in }}=$ $10^{-18} \mathrm{~m}^{2}$ were used [11].

In this study, $\mu=\mu_{W}=\mu_{V}=\mu_{P}$ was assumed and was set to $\mu=10^{-3} \rho_{\mathrm{s}}^{2} \Omega_{\text {ci }}$. This is a rough estimation of the viscosity, and the dependence of instability on viscosity is discussed in $\$ 4-4$.

\subsection{Unstable mode}

Figure 3 (a) shows the growth rate as a function of the azimuthal and axial mode numbers $m$ and $n$, respectively, using the parameters given in the previous subsection. There are some unstable modes, and the most unstable mode was found to be $(m, n)=(2,2)$. The growth rate and eigen frequency for $(m, n)=(2,2)$ were found to be $5 \times 10^{2} \mathrm{~s}^{-1}$ and $12 \mathrm{kHz}$, respectively. However, modes with larger $m$ and $n$ values were found to be stable, and mode $(m, n)=(1,1)$ was also stable in this case. Figure $3(\mathrm{~b})$ shows the mode structure of the ion pressure for $(m, n)=$ $(2,2)$. Modes excited in this simulation had a maximum at the $r=25 \sim 30 \mathrm{~mm}$ and no node in the plasma.

\subsection{Parameter dependence}

The dependence of the growth rate on the plasma parameters was investigated. The parameters we studied were the magnetic field $B$, the device length $\lambda$, the gradient lengths $L_{N}$ and $L_{T \mathrm{i}}$, and temperatures $T_{\mathrm{e}}$ and $T_{\mathrm{i} 0}$, which

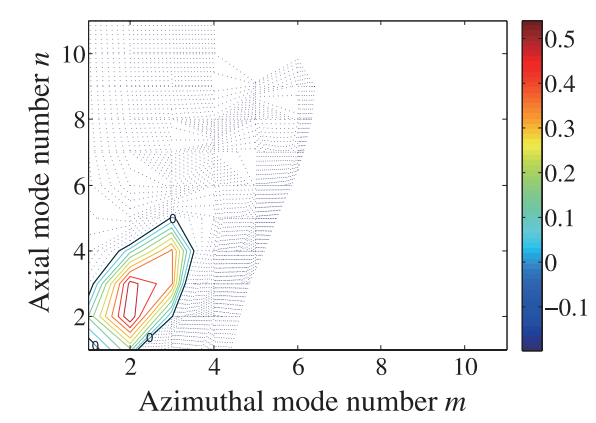

(a)

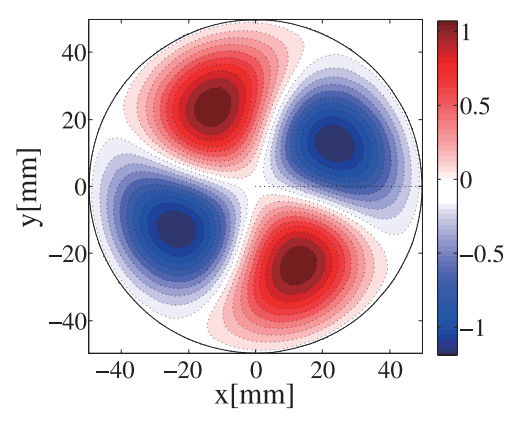

(b)

Fig. 3 (a): Growth rate as a function of the azimuthal and axial mode numbers. Solid and dotted lines represent unstable and stable contours, respectively. (b): Mode structure of the ion pressure with $(m, n)=(2,2)$, which is the most unstable. excite ITG modes in the PANTA. These dependencies can be compared with the analytical solutions found in $\S 3$.

Figure 4 (a) shows the growth rate for the $(m, n)=$ $(2,2)$ as a function of different gradient lengths. The unstable region for different gradient lengths was limited, and the dependence on that of density $L_{N}$ is strong. Figure 4 (b) shows the growth rate for the $(m, n)=(2,2)$ as a function of different temperatures, indicating that lower temperatures are preferable for excitation. The effect of dissipations, which make modes stable at higher temperatures, was found to be strong, and therefore, provides the upper temperature limits.

Figure 5 (a) shows the unstable region in the $B-a$ space. There was no unstable mode found for $B \sim 0.1 \mathrm{~T}$, which is the maximum magnetic field in the PANTA experiments. Figure 5(b) shows the unstable region in the $\lambda-a$ space. There is no unstable mode for shorter device lengths $(\lambda)$. The dependence of the growth rate on $\lambda$ was weaker than for other plasma parameters. The parameter $\rho_{\mathrm{s}} k_{\perp}$ is inversely proportional to $a$ and $B$ : thus, the unstable region is limited in the $B-a$ space. The parameter $k_{z}$ is inversely proportional to $\lambda$, and the unstable region is also limited in the $\lambda-a$ space.

Summarizing the results of the parameter scans, we found that excitation conditions are strongly limited by the ion temperature $T_{\mathrm{i} 0}$ and the density gradient length $L_{N}$.

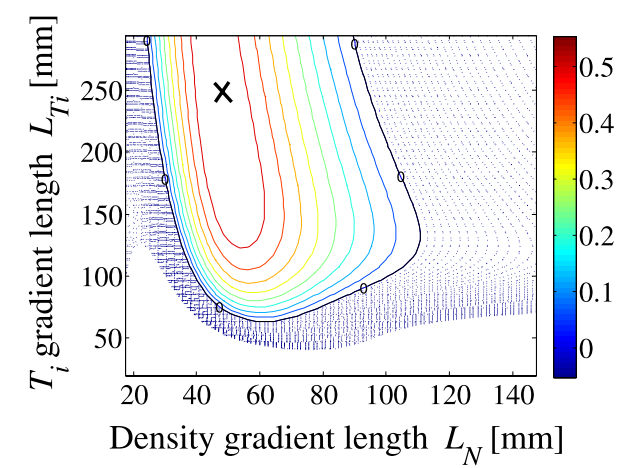

(a)

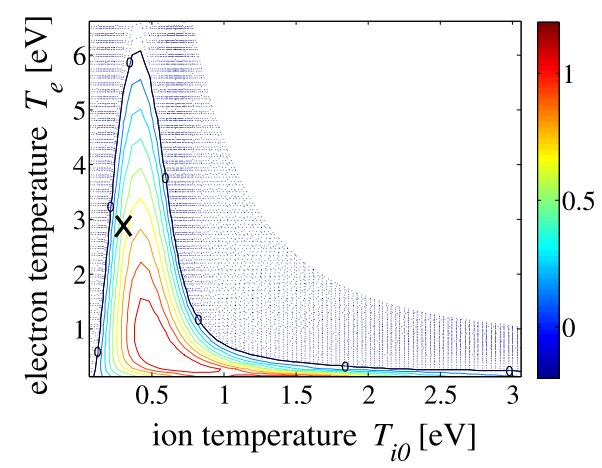

(b)

Fig. 4 Growth rate as a function of (a) gradient lengths $L_{N}$ and $L_{T \mathrm{i}}$ and (b) temperatures $T_{\mathrm{e}}$ and $T_{\mathrm{i} 0}$. The cross mark shows a typical parameter of the PANTA experiments. 


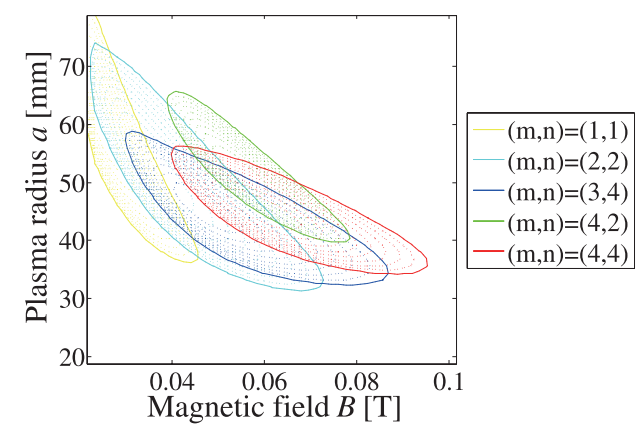

(a)

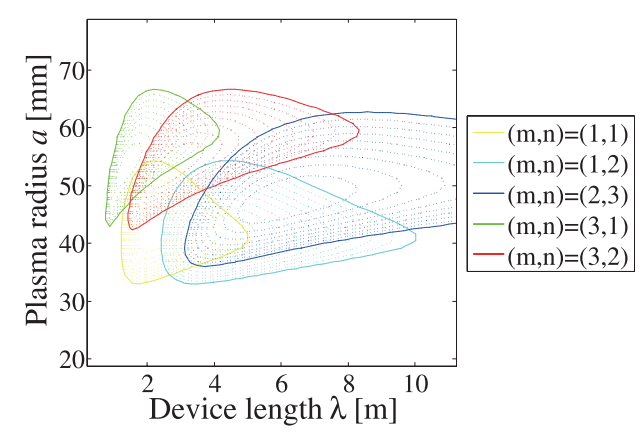

(b)

Fig. 5 Unstable regions in the (a) $B-a$ space and (b) $\lambda-a$ space. A total of 5 unstable modes are plotted.

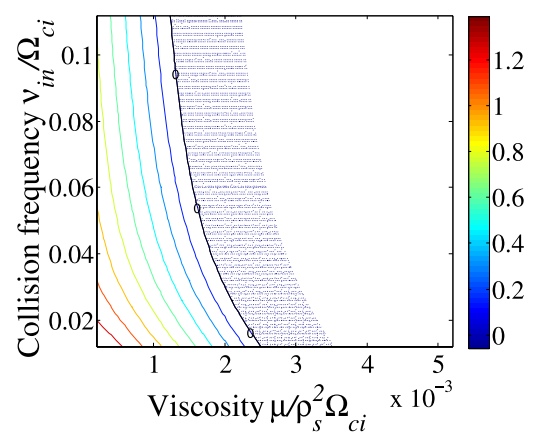

Fig. 6 Growth rate as a function of the ion-neutral collision frequency and viscosity.

\subsection{Effect of collisions}

The effects of collisions and dissipations on stability are described here. Figure 6 shows the growth rate for $(m, n)=(2,2)$ as a function of an ion-neutral collision frequency and viscosity. We found that modes were stabilized with larger ion-neutral collision frequencies and viscosi- ties, and the dependence of the growth rate on viscosity was strong. Overall, the excitation of ITG modes in the PANTA depends on the actual viscosity.

\section{Summary}

To investigate turbulent plasmas by identifying the excitation conditions of ITG modes in the PANTA, linear growth rates of ITG modes were analyzed to determine its excitation conditions. A three-field model, including potential, parallel flow of ions, and ion pressure which also account for the effect of neutrals, was used for the analysis. Parameter scans showed the thresholds for ITG mode excitation, which were found to strongly depend on the ion temperature $T_{\mathrm{i} 0}$ and the density gradient length $L_{N}$. We also estimated the effects of collisions and dissipations on the growth rate in this study. Futermore, the dependences for instability can be compared with the analytical solutions. Our analyses show that it is possible to excite ITG modes in the PANTA.

The dependence of the growth rate on density and temperature profiles was not considered in this paper, though they do affect the instability [12]. Future studies will involve analyses of these profiles and the nonlinear saturation of ITG modes.

\section{Acknowledgments}

This study is supported by a Grant-in-Aid for Scientific Research $(21224014,23360414)$ from JSPS, and by the collaboration program of NIFS (NIFS10KOAP023, NIFS11KNST013, NIFS12KNTT012) and of RIAM of Kyushu University.

[1] W. Horton, Rev. Mod. Phys. 71, 735 (1999).

[2] Z. Lin et al., Science 281, 1835 (1998).

[3] W.W. Lee and W.M. Tang, Phys. Fluids 31, 612 (1988).

[4] A.K. Sen et al., Phys. Rev. Lett. 66, 429 (1991).

[5] S.E. Parker and A.K. Sen, Phys. Plasmas 9, 3440 (2002).

[6] S. Oldenbürger et al., Plasma Phys. Control. Fusion 54, 055002 (2012).

[7] N. Ezumi et al., J. Nucl. Mater. 337, 1106 (2005).

[8] S. Hamaguchi and W. Horton, Phys. Fluids B 2, 1833 (1990).

[9] N. Kasuya et al., Phys. Plasmas 15, 052302 (2008).

[10] R. Balescu, Aspects of Anomalous Transport in Plasmas (IOP Publishing, Bristol, 2005) p. 55.

[11] A.V. Phelps, J. Phys. Chem. Ref. Data 20, 557 (1991).

[12] T.S. Hahm and W.M. Tang, Phys. Fluids B 1, 1185 (1989). 